\title{
A novel structure of photonic crystal fibre for dispersion compensation over broadband range
}

\author{
MADHAVI WAGHMARE* and K T V REDDY \\ Department of Electronics and Telecommunication Engineering, Sardar Patel Institute of Technology, \\ University of Mumbai, Mumbai 400 058, India \\ e-mail:01madhavi@gmail.com; ktvreddy@gmail.com
}

MS received 14 June 2016; revised 4 April 2017; accepted 17 April 2017; published online 23 October 2017

\begin{abstract}
This paper studies a novel structure of photonic crystal fibre (PCF) for dispersion compensation at broadband wavelengths. The application of broadband is investigated using a design model based on combination of modal properties and dispersion compensation. The newly designed PCF with defect introduced is recorded over transmission spectrum range 146.7-256.98 THz, i.e., 1.16-2.04 $\mu \mathrm{m}$. The modal characteristics and dispersion compensation of 2D PCF with circular air holes defect introduced are investigated and compared to those of conventional hexagonal 2D PCF. Changes in bandwidth behaviour are also observed by changing refractive index and geometric parameter of PCF.
\end{abstract}

Keywords. Dispersion compensation; photonic crystal fibre; refractive index.

\section{Introduction}

The development of transmission rate experiences restrictions due to dispersion in optic fibre communication field. The best method for reduced dispersion in broadband of wavelength is to use the dispersion compensation method. The single-mode fibre usually has positive dispersion and this dispersion increases while propagating over long distance. The photonic crystal fibre (PCF) structure needs to be designed in order to reduce positive dispersion and this can be achieved by inserting dispersion-compensating PCF with negative dispersion in the transmission link. In the conventional PCF, results of negative dispersion have been studied, where $D=-100 \mathrm{ps} / \mathrm{nm} / \mathrm{km}$ at $1550 \mathrm{~nm}$ wavelength with high losses [1]. The modal characteristics of PCFs, with guiding cores consisting of one or seven missing air holes, are investigated using finite-element method and compared to those of conventional fibres. For broadband in PCF there are several designs, one of which is a double-clad fibre, which has been investigated to enhance the output power [2]. Another method to enhance output power is dispersion compensation of PCF for high-speed transmission systems [3].

For high-speed and large-bandwidth application in optic fibre communication, the dispersion characteristic requires flattened dispersion. The dispersion characteristic should be uniform or should have minimum slope throughout the wavelength range by an optimized technique. For this method, investigation was carried out using a genetic

*For correspondence algorithm to minimize pulse broadening at large wavelengths [4]. The microstructure of PCF can be used for residual dispersion compensation. A flattened negative dispersion was proposed by Varshney et al [5] for residual dispersion compensation. With this microstructure, ultraflattened negative dispersion of $-98.3 \mathrm{ps} / \mathrm{nm} / \mathrm{km}$ and $\Delta D=1.1 \mathrm{ps} / \mathrm{nm} / \mathrm{km}$ over $\mathrm{S}-\mathrm{L}$ wavelength band, i.e., wavelength bandwidth of $1.48-1.63 \mu \mathrm{m}$, were reported. Another microstructure used for residual dispersion compensation yielded ultra-flattened negative dispersion of $-179 \mathrm{ps} / \mathrm{nm} / \mathrm{km}$ and $\Delta D=2.1 \mathrm{ps} / \mathrm{nm} / \mathrm{km}$ over $\mathrm{S}-\mathrm{U}$ wavelength band, i.e., wavelength bandwidth of $1.48-1.675 \mu \mathrm{m}$ [6]. The design by Silva et al [7] shows higher average dispersion of $-212 \mathrm{ps} / \mathrm{nm} / \mathrm{km}$ and $\Delta D=11.9 \mathrm{ps} / \mathrm{nm} / \mathrm{km}$ over E-U wavelength band, i.e., wavelength bandwidth of 1.35-1.7 $\mu \mathrm{m}$, and the design has been studied with Ge-doped core using a genetic algorithm. In the conventional hexagonal PCF, a structure with missing central hole is considered and compared to the one with air holes defect introduced in PCF. The number of defects is the number of circular holes at the centre of PCF. By this method, absolute dispersion variation is $-227 \mathrm{ps} / \mathrm{nm} / \mathrm{km}$ with $\quad \Delta D \sim 11 \mathrm{ps} / \mathrm{nm} / \mathrm{km}$ over frequency range 178.98-202.56 THz with the design of equiangular spiral PCF structure [8]. The design of PCF structure was studied for residual dispersion compensation of $-457.4 \mathrm{ps} / \mathrm{nm} / \mathrm{km}$ over the range larger than E-U band, i.e., $177.3-220 \mathrm{THz}$, and $\Delta D \sim 11.9 \mathrm{ps} / \mathrm{nm} / \mathrm{km}$ [9]. The results of applicability of PCF with uniform air hole structure to high-speed and broadband transmission over conventional telecommunication bands have been considered. However, it is difficult 
to compensate for dispersion and achieve zero loss over a broadband range with PCF structure.

More flattened negative dispersion leads to good dispersion compensation and almost zero confinement loss, over enhanced bandwidth. The proposed structure is designed to compensate dispersion of light propagation and achieve nearly zero confinement loss. In this structure, inner circular air hole structure is used to reduce dispersion.

This paper discusses the effect of defect introduction in the novel designed PCF in the transmission spectrum range 146.7-256.98 THz with $-533.74 \mathrm{ps} / \mathrm{nm} / \mathrm{km}$ average large negative dispersion.

The biggest advantage of using this novel structure over conventional fibres is the possibility of obtaining higher ultra-flattened negative dispersion over a broadband range.

\section{Modelling of a defect in PCF}

PCF has air hole structures of two different forms, one is of square shape and the other is triangular. PCFs have attracted more attention because of their special characteristics with triangular shape of air holes [10]. The idea in the proposed structure of using hexagonal PCF with inner circular air holes comes after studying two different reports. One report discussed about conventional hexagonal PCF [11] and the other about circular PCF [8]. The authors have tried to combine designs of both structures from these reports to produce a new structure design combining hexagonal PCF structure with inner circular air holes. The introduction of circular air holes causes light to be confined between circular air holes and hexagonal PCF. When the PCF structure has inner (core region) and outer (cladding region) structures together, then effective refractive index can be adjusted by changing geometric parameters of inner and outer regions. Chromatic dispersion can be affected by the large effective refractive index difference between inner and outer region [13]. Also, the symmetry of the structures prevents the existence of a linear electro-optic effect [14]. The core (inner) region produces better negative dispersion. The number of air hole rings in core region is chosen such that it helps in achieving large negative dispersion, while the number of air hole rings in the outer region is selected such that it helps in achieving very low confinement loss. Figure 1 shows a cross section of the newly designed PCF structure in the $x-y$ plane. The inner circular air holes with diameter $d$ and outer hexagonal structure are arranged with $a$ as the pitch, i.e., distance between holes, and $r$ is radius of air holes. The values of $r, a$ and diameter $d$ of PCF structure are simulated to obtain the flattened negative dispersion for broadband spectrum. The number of air hole rings of hexagonal PCF is fixed to $N=5$, in which inner three air hole hexagonal rings are replaced with circular rings. The placing of air holes is optimized to get better output. The number of air holes of outer region gives almost zero confinement loss [9].

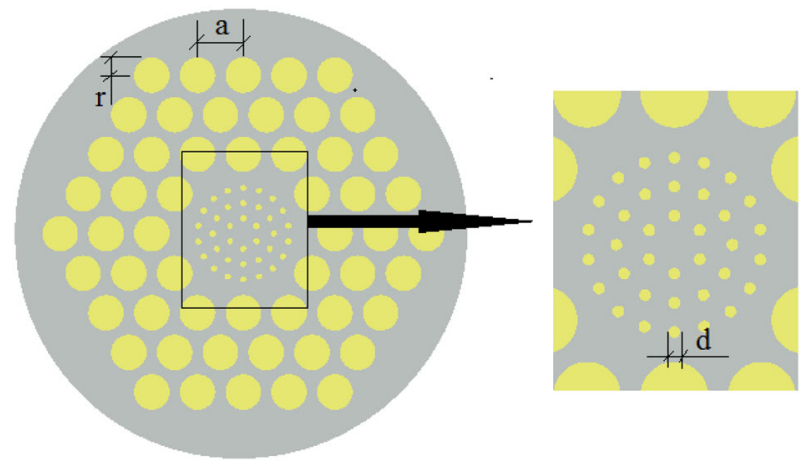

Figure 1. PCF with defect at the centre.

A commercially available Finite-Difference Time Domain (FDTD) software (Lumerical) was used to evaluate the proposed structure. The wavelength-dependent refractive index of the silica was included in the simulation through the Sellmeier equation.

The chromatic dispersion $D$ in $\mathrm{ps} / \mathrm{nm} / \mathrm{km}$ of a PCF is easily evaluated from the $\eta_{\text {eff }}$ value variation vs. the wavelength using the following equation:

$$
D=-\frac{\lambda d^{2} \operatorname{Re}\left[\eta_{e f f}\right]}{c}
$$

where $c$ is the speed of light in vacuum and $\operatorname{Re}\left[\eta_{\text {eff }}\right]$ is the real part of the refractive index. In PCFs, the chromatic dispersion $D$ is also related to the other design parameters like geometry of the air holes, pitch and inner circular holes diameter. By optimizing these parameters, suitable guiding properties can be obtained [10].

Confinement loss $L_{c}$ is the light confinement ability within the core region. The increase of number of air hole rings supports the confinement of light in the core region, which results in minimum losses than those with less air hole rings. Also, increasing the air hole radius results in the increase of the air filling fraction and accordingly decreases the loss.

$$
L_{c}=\frac{\left(20 \times 10^{6}\right)}{\ln (10)} K_{0} \operatorname{Im}\left[\eta_{e f f}\right]
$$

where $K_{0}=2 \pi / \lambda$ is wave number in free space with the unit $\mathrm{dB} / \mathrm{m}$ and $\operatorname{Im}\left[\eta_{\text {eff }}\right]$ is the imaginary part of the refractive index. From Eqs. (1) and (2), it is observed that the dispersion is dependent on variation of real value of refractive index with wavelength and confinement loss is dependent on imaginary part of refractive index [12]. The relation between refractive index and group velocity is

$$
v_{\mathfrak{g}}=\left(\frac{\partial \mathcal{K}}{\partial \omega}\right)^{-1}=c \frac{\partial}{\partial \omega}\left(\omega \eta_{e f f}\right)^{-1}=\frac{\mathrm{c}}{\eta_{e f f}+\omega \frac{\partial \eta}{\partial \omega}}=\frac{\mathrm{c}}{\eta_{g}(\omega)}
$$


where $\eta_{\text {eff }}$ is the refractive index and $\eta_{g}$ is the group index. The wavenumber $k$ can be considered as the change in spectral phase per unit length [15].

\section{Results and analysis}

The design of PCF structure should be such that output is optimized, i.e., more negative dispersion and confinement loss equal to almost zero. Optimum parameters were obtained by changing various combinations of PCF parameters. Finally, by varying one of the parameters of the structure, the results reached the optimum value as compared with the previous best result, for broader frequency spectrum range [9]. The novel design provides better results than the previously carried out research works.

In general, the decrease in pitch $a$ or $d$ of a PCF leads to increase in negative dispersion. Increase in negative dispersion due to increase in $r$ indicates the reduction in effective refractive index.

It was observed that the modes profile of PCF having circles in hexagon structure is more confined around outer region of PCF core. This is due to relatively high refractive index of the cladding region as compared with lower refractive index of core region.

Parameters in the new design were optimized to the following: inner circular air hole diameter $d=0.055 \mu \mathrm{m}$, outer hexagonal structure pitch value $a=0.3 \mu \mathrm{m}$ and radius of outer hexagonal structure $r=0.1 \mu \mathrm{m}$.

The proposed novel structure shows large average dispersion range from -481.041 to $-546.43 \mathrm{ps} / \mathrm{nm} / \mathrm{km}$ and in the spectrum range 146.7-256.98 THz, i.e., 1.166-2.04 $\mu \mathrm{m}$. Figure 2 shows the large spectrum range coverage for dispersion compensation. The optimum parameter gives high average dispersion compensation with broadband spectrum.

Figure 3 shows that the new design structure records transmission range $1.1621-2.044 \mu \mathrm{m}$, i.e., $146.7-220 \mathrm{THz}$,

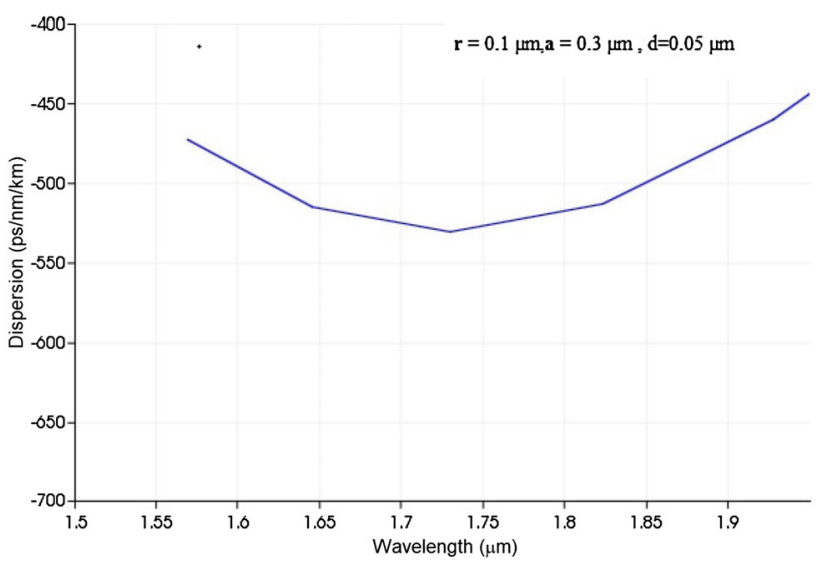

Figure 2. Flattened negative dispersion at optimum parameter with wavelength varying from 1.57 to $2 \mu \mathrm{m}$.

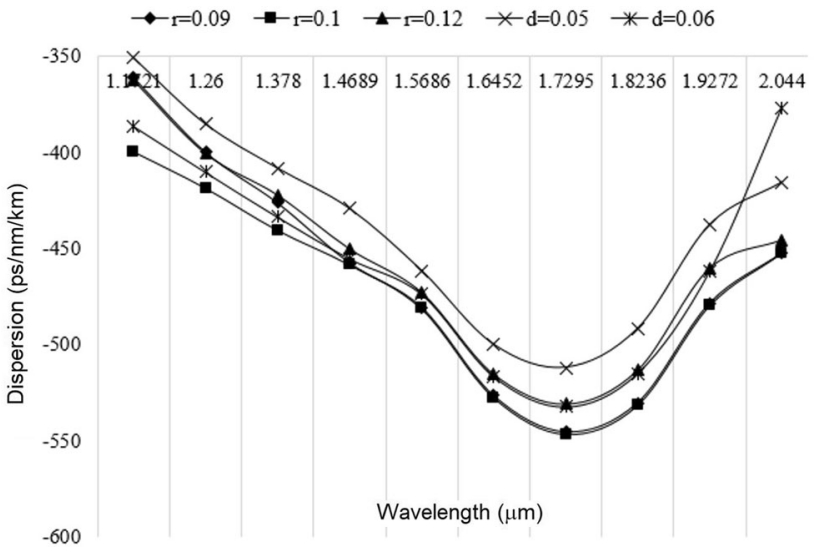

Figure 3. Variation of dispersion Variation with pitch $a$ and radius $r$ of the outer hexagonal structure in the spectrum wavelength range $1.17-2.04 \mu \mathrm{m}$. (a)

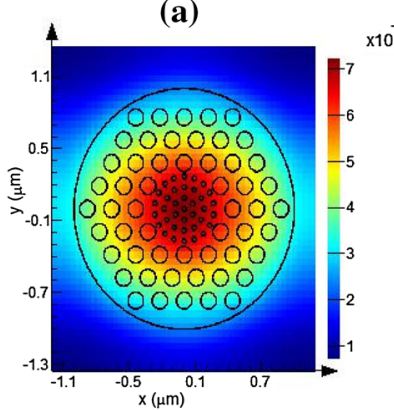

(b)

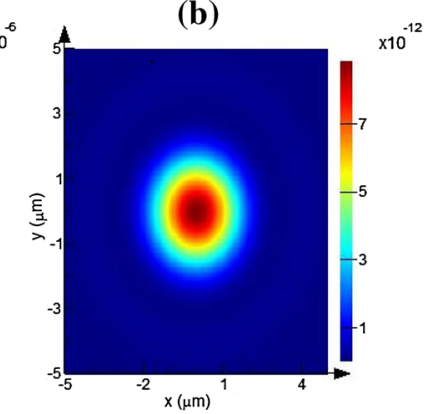

Figure 4. (a) Simulated LP01 mode profile of proposed structure and (b) Gaussian energy density of proposed structure.

Table 1. Numerical analysis results.

\begin{tabular}{lc}
\hline Parameters $(\mu \mathrm{m})$ & Dispersion $(\mathrm{ps} / \mathrm{nm} / \mathrm{km})$ \\
\hline$r=0.09, d=0.055$ & -544.437 \\
$r=0.10, d=0.055$ & -533.74 \\
$r=0.12, d=0.055$ & -532.260 \\
$r=0.10, d=0.05$ & -511.908 \\
$r=0.10, d=0.06$ & -532.264 \\
\hline
\end{tabular}

For all these geometric parameters: spectrum range $(\mu \mathrm{m})=1.17-2.044$ confinement loss $L_{c}(\mathrm{db} / \mathrm{cm})=0, \Delta D(\mathrm{ps} / \mathrm{nm} / \mathrm{km})= \pm 12$ and bandwidth $(\mathrm{nm})=874$.

with $-533.74 \mathrm{ps} / \mathrm{nm} / \mathrm{km}$ average large negative dispersion. This is larger bandwidth than previous results. In this spectrum range, more flattened negative dispersion can be calculated for $1.568-2.044 \mu \mathrm{m}$; this result is shown in figure 5 .

Figure 4a shows average Poynting vector profile in propagation direction and figure $4 \mathrm{~b}$ shows Gaussian energy density of proposed structure for both of the fundamental degenerate modes. It can also be observed that the modes 
Table 2. Comparison of results.

\begin{tabular}{lcccc}
\hline References & $\begin{array}{c}\text { Spectrum } \\
\text { range }(\mu \mathrm{m})\end{array}$ & $\begin{array}{c}\text { Dispersion }(\mathrm{ps} / \\
\mathrm{nm} / \mathrm{km})\end{array}$ & $\begin{array}{c}\Delta D(\mathrm{ps} / \\
\mathrm{nm} / \mathrm{km})\end{array}$ & $\begin{array}{c}\mathrm{BW} \\
(\mathrm{nm})\end{array}$ \\
\hline$[5]$ & $1.48-1.63$ & -98.3 & $\sim 1.1$ & 150 \\
{$[6]$} & $1.48-1.675$ & -179 & 2.1 & 195 \\
{$[7]$} & $1.35-1.7$ & -212 & 11.9 & 350 \\
{$[8]$} & $1.35-1.675$ & -227 & 11 & 325 \\
{$[9]$} & $1.36-1.69$ & -457.4 & 11.9 & 330 \\
Proposed & $1.16-2.04$ & -533.74 & 12 & 874 \\
\hline
\end{tabular}

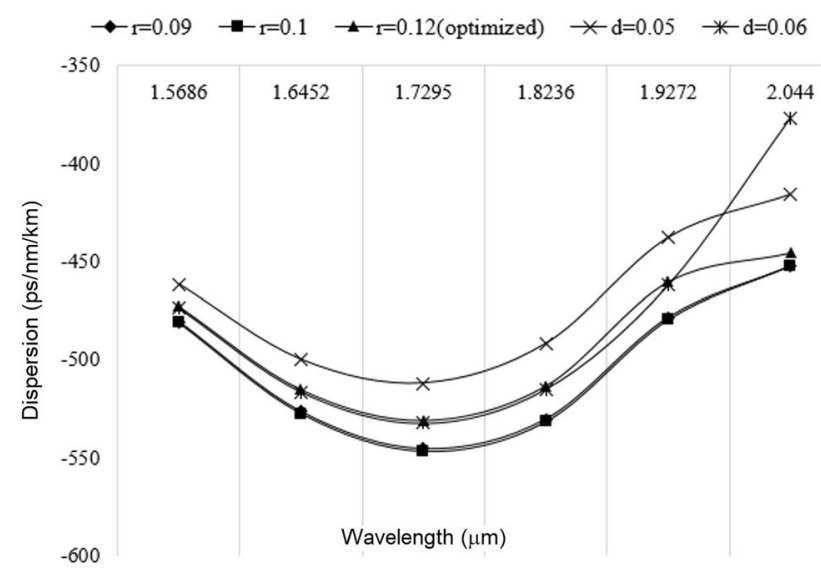

Figure 5. Sensitivity of the dispersion for $\pm 9 \%$ variation in pitch $a$ and radius $\boldsymbol{r}$ of the outer hexagonal structure with wavelength varying from 1.57 to $2 \mu \mathrm{m}$.

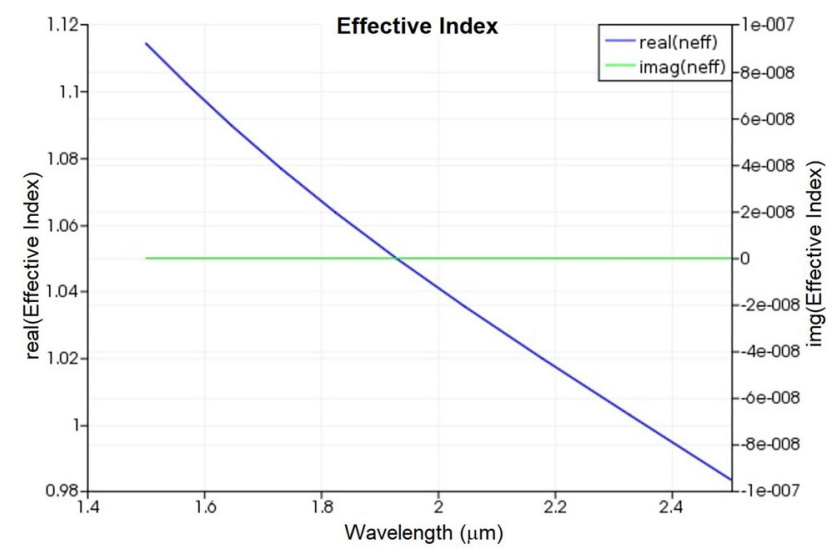

Figure 6. Effective index at optimum parameter.

are confined in outer region of circular air holes and refractive index is higher in the outer region of circular air holes as compared with the central region of the circular air holes.

Table 1 shows the results of the numerical analysis carried out on the proposed PCF structure with variation in geometric parameters. (a)

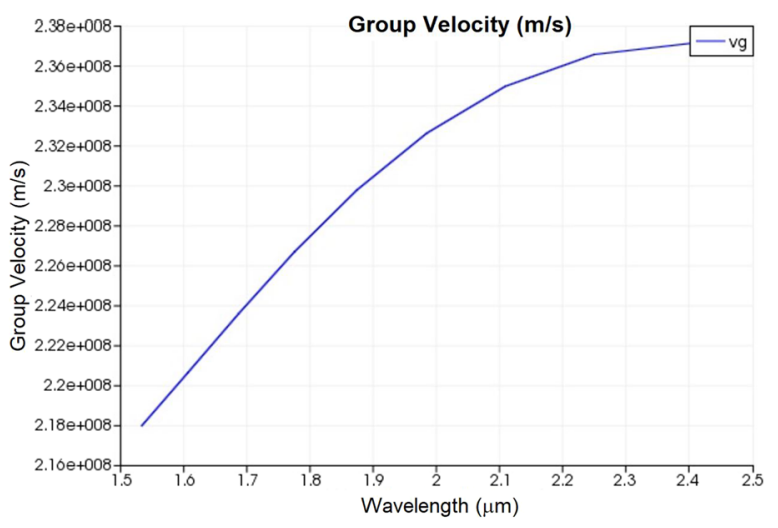

(b)

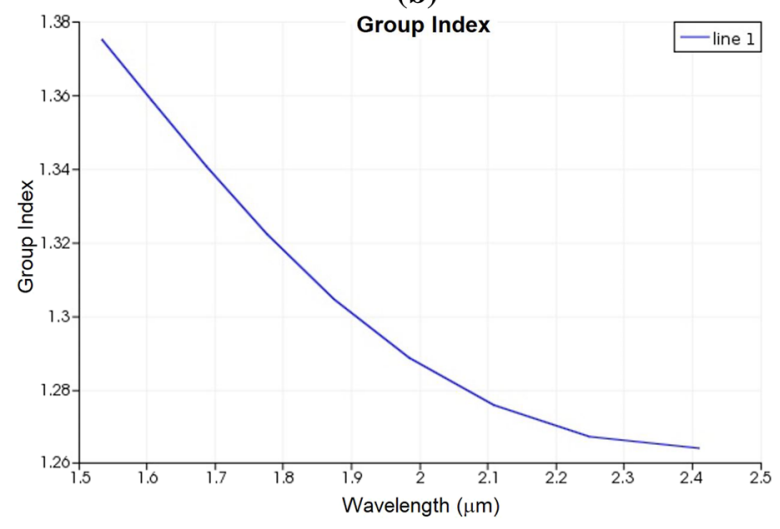

Figure 7. (a) Group velocity and (b) group index at optimum parameter.

Table 2 shows comparison of results from the proposed structure with the previous results. Results obtained for the proposed structure are superior when compared with earlier results.

Figure 5 shows that change in inner air hole diameter $d$, pitch $a$ and radius $r$ of outer air hole changes chromatic dispersion of the new design PCF structure. It also shows the sensitivity of dispersion for $\pm 9 \%$ variation in pitch $a$ and the radius $r$ of the outer hexagonal structure.

In figure 6, real part of effective index indicates that there is linear dispersion of light propagation and imaginary part indicates that the confinement loss is almost zero, i.e., confinement is better at the centre of the PCF from Eqs. (1) and (2).

For light propagating in $\mathrm{PCF}$, the group velocity can be calculated by substituting the effective refractive index for the refractive index $\eta$ in Eq. (3). The deviation in the result from group velocity (Eq. 3) in PCF structurecan be interpreted as the influence of waveguide dispersion. The group velocity of light (shown in figure $7 \mathrm{a}$ and $\mathrm{b}$ ) in a medium is defined as the inverse of the derivative of the wavenumber with respect to angular frequency. 
The fabrication of the novel structure can be achieved through a standard assembling process to speed up fibre drawing work in the near future [16].

\section{Conclusion}

After the thorough numerical analysis of the proposed structure, we can conclude that this can be used for broadband communication in the spectrum range 128.49-256.98 THz, i.e., 1.1621-2.044 $\mu \mathrm{m}$. The novel design structure shows the average dispersion of $-533.74 \mathrm{ps} / \mathrm{nm} / \mathrm{km}$ with flattened dispersion from -481.041 to $-546.43 \mathrm{ps} / \mathrm{nm} / \mathrm{km}$ over $884 \mathrm{~nm}$ spectrum range. As compared with the conventional $\mathrm{PCF}$, this result is 2.5 times higher than the previous best results.

\section{Acknowledgements}

The authors wish to thank the anonymous reviewers for their useful comments and constructive suggestions. The authors would like to thank Sardar Patel Institute of Technology Research Centre, Mumbai, India, for simulation.

\section{References}

[1] Begum F, Namihira Y, Razzak S M A, Kaijage S, Hai N H, Kinjo T, Miyagi K and Zou N 2009 Novel broadband dispersion compensating photonic crystal fiber: application in high-speed transmission systems. Opt. Laser Technol. 41: 679-686

[2] Joannopoulos J D, Meade R D and Winn J N 1995 Photonic crystal: molding the flow of light. Princeton University Press. pp. 232-233

[3] Begum F, Namihira Y, Kaijage S F, Razzak S M A, Hai N H, Kinjo T, Miyagi K and Zou N 2009 Design of broadband dispersion compensating photonic crystal fibers for high speed transmission systems. In: Proceedings of the IEEE Conference on OSA OFC

[4] Makoui S, Savadi-Oskouei M, Rostami A and Kanani Z D K 2009 Dispersion flattened optical fiber design for large bandwidth and high-speed optical communications using optimization technique. Prog. Electromagn. Res. B 13: $21-40$
[5] Varshney S K, Florous N J, Saitoh K, Koshiba M and Fujisawa T 2007 Numerical investigation and optimization of a photonic crystal fiber for simultaneous dispersion compensation over $\mathrm{S}+\mathrm{C}+\mathrm{L}$ wavelength bands. Opt. Commun. 274: 74-79

[6] Franco M A R, Serrao V A and Sircilli F 2008 Microstructured optical fiber for residual dispersion compensation over $\mathrm{S}+\mathrm{C}+\mathrm{L}+\mathrm{U}$ wavelength bands. IEEE Photon. Technol. Lett. 20(9): 751-753

[7] da Silva J P, Bezerra D S, Rodriguez-Esquerre V F, da Fonseca I E and Hernández-Figueroa H E 2010 Ge-doped defect-core microstructure fiber design by genetic algorithm for residual dispersion compensation. IEEE Photon. Technol. Lett. 22(18): 1337

[8] Islam M A and Alam M S 2012 Design of a polarizationmaintaining equiangular spiral photonic crystal fiber for residual dispersion compensation over $\mathrm{E}+\mathrm{S}+\mathrm{C}+\mathrm{L}+\mathrm{U}$ wavelength bands. IEEE Photon. Technol. Lett. 24(11): 930-932

[9] Tee D C, Abu Bakar M H, Tamchek N and Mahamd Adikan F R 2013 Photonic crystal fiber in photonic crystal fiber for residual dispersion compensation over $\mathrm{E}+\mathrm{S}+\mathrm{C}+\mathrm{L}+\mathrm{U}$ wavelength bands. IEEE Photon. Technol. Lett. 5(3): 1-10. ISSN: 1943-0655

[10] Waghmare M H and Reddy K T V 2014 Analysis of photonic bandgap structure in 2D hexagonal and square lattice structure of photonic crystal. In: Proceedings of ICACACT-2014, IEEE Explore Digital Library

[11] Li H, Mafi A, Schülzgen A, Li L, Temyanko V L, Peyghambarian N and Moloney J V 2007 Analysis and design of photonic crystal fibers based on an improved effective-index method. J. Lightw. Technol. 25(5): 1224-1230

[12] Begum F, Namihira Y, Razzak S M A, Kaijage S, Hai N H, Kinjo T, Miyagi K and Zou N 2009 Novel broadband dispersion compensating photonic crystal fibers: applications in high-speed transmission systems. Opt. Laser Technol. 41: 679-686

[13] Agrawal G P 2002 Fiber-optic communication systems, 3rd ed. New York, NY, USA: Wiley, pp. 15-64

[14] Waghmare M H and Reddy K T V 2016 Design and modal analysis of photonic crystal fiber for dispersion compensation over broadband range. J. Microw. Optoelectron. Electromagn. Appl. 15(4): 365-379

[15] R.P. Photonics buyer's guide. https://www.rp-photonics.com/ buyersguide.html

[16] Knight J C, Birks T A, Russell P S J and Atkin D M 1996 All-silica single-mode optical fiber with photonic crystal cladding. Opt. Lett. 21(19): 1547-1549 